\title{
Mit der MMW in die e.Akademie
}

- Sie halten das erste CME-Sonderheft 2013 der MMW-Fortschritte der Medizin in Händen. Damit können Sie bequem am häuslichen PC 24 CME-Punkte sammeln. Wir haben uns wieder bemüht, das breite medizinische Spektrum der hausärztlichen Tätigkeit abzubilden: Die Themen reichen von der Osteoporosetherapie über die Anämieabklärung bis zum richtigen Umgang mit dem Praxisproblem Reizdarm.

Sie finden nach jeder der zertifizierten Fortbildungseinheiten einen Fragebogen, den Sie online in der e.Akademie von Springer Medizin ausfüllen können. Die e.Akademie ist mit über 600 zertifizierten Kursen aus allen medizinischen Fachgebieten - darunter aktuell 350 aus der Inneren und Allgemeinmedizin - das umfangreichste Online-Fortbildungsangebot in Deutschland. Teilnehmen und Punkte sammeln können Sie
- als e.Med-Abonnent uneingeschränkt an allen Kursen der e.Akademie,

- als Abonnent einer Fachzeitschrift an den Kursen der abonnierten Zeitschrift oder - an ausgewählten Kursen dieser Sonderausgabe der MMW bis zum 11. April 2013 kostenlos unter Verwendung der Fortbildungs-Identifikations-Nummer (FIN), die auf den Fragebögen aufgedruckt ist.

Mit der FIN erhalten Sie einen kleinen Einblick in das große Angebot der e.Akademie, die wiederum nur ein kleiner Teil des e.Med-Paketes von Springer Medizin ist. e.Med eröffnet Ärzten einen umfassenden Zugang zur digitalen Welt der Medizin: Fortbilden, CME-Punkte sammeln, recherchieren, täglich aktuelle Nachrichten aus Medizin und Gesundheitspolitik, Expertenbzw. Kollegenrat einholen - das alles und noch mehr bietet e.Med. Dabei sind alle Bausteine so konzipiert, dass sie Ärzte in ih-

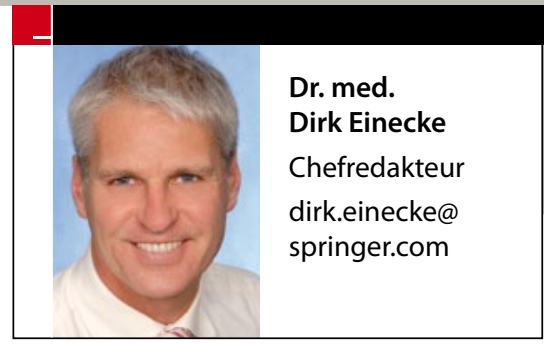

rem Berufslltag bestmöglich unterstützen. Mit einem Probeabo können Sie e.Med 30 Tage lang kostenlos und unverbindlich testen und die e.Med-Welt entdecken.

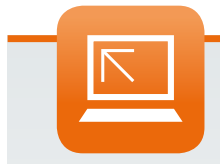

Weitere Infos auf springermedizin.de

Zur e.Akademie geht es auf www.springermedizin.de/infoeakademie

Direkt zu den Kursen der MMW gelangen Sie über $>$ www. springermedizin.de/kursemmw Ihr Probeabo bei e.Med können Sie unter $>$ www.springermedizin.de/eMed ordern.

\section{RAUCHSTOPP}

\section{Keine Angst vor der Gewichtszunahme!}

Wer mit dem Rauchen aufhört, senkt sein kardiovaskuläres Risiko, auch wenn er etwas an Gewicht zulegt. In einer prospektiven Kohortenstudie mit mehr als 3200 Teilnehmern über 25 Jahre analysierten Bostoner Wissenschaftler die Assoziation zwischen einer Gewichtszunahme über vier Jahre nach dem Rauchstopp und der Inzidenz kardiovaskulärer Ereignisse bei Erwachsenen mit und ohne Diabetes.

Insgesamt 631 kardiovaskuläre Ereignisse wurden dokumentiert. Im Mittel nahmen diejenigen, die mit dem Rauchen aufgehört hatten, über den Zeitraum von vier Jahren $1,4 \mathrm{~kg}$ an Gewicht zu.
Im Vergleich zu Rauchern war das kardiovaskuläre Risiko bei Teilnehmern, die mit dem Rauchen aufgehört hatten, signifikant um 50\% Das lohnt sich auf jeden Fall! verringert. Bei Nie-

Rauchern war es im Vergleich zu Rauchern um $68 \%$ verringert. Ähnliche Werte ergaben sich bei Diabetikern und bei Berücksichtigung von kardiovaskulären Risikofaktoren und Gewichtsveränderungen.

Clair C et al. JAMA 2013; 309(10): 1014-1021; DOI: 10.1001/jama.2013.1644.

\section{DEUTLICHE RISIKOREDUKTION}

\section{ASS gegen Melanom?}

Acetylsalicylsäure (ASS) senkte in einer großen US-Studie das Risiko für ein malignes Melanom. Auf der Grundlage von Daten der Women's Health Initiative wurden fast 60000 hellhäutige, postmenopausale Frauen über rund zwölf Jahre nachverfolgt. Frauen, die ASS einnahmen, hatten im Vergleich zu Frauen, die keine nicht steroidalen Antirheumatika schluckten, ein um 21\% niedrigeren Melanomrisiko. Je länger die Frauen ASS genommen hatten, desto besser waren sie offenbar geschützt. Nach fünf Einnahmejahren betrug die Risikoreduktion bereits $30 \%$.

Cancer 2013, online 11. März; doi: 10.1002/cncr.27817 